\title{
External quality assessment of malaria microscopy in the Democratic Republic of the Congo
}

\author{
Pierre Mukadi ${ }^{1}$, Philippe Gillet ${ }^{2 *}$, Albert Lukuka ${ }^{1,3}$, Ben Atua ${ }^{3}$, Simelo Kahodi ${ }^{4}$, Jean Lokombe ${ }^{1,5}$, \\ Jean-Jacques Muyembe $e^{1,5}$ and Jan Jacobs ${ }^{2,6}$
}

\begin{abstract}
Background: External quality assessments (EQA) are an alternative to cross-checking of blood slides in the quality control of malaria microscopy. This study reports the findings of an EQA of malaria microscopy in the Democratic Republic of the Congo (DRC).

Methods: After validation, an EQA slide panel and a questionnaire were delivered to diagnostic laboratories in four provinces of DRC. The panel included three samples for diagnosis (sample 1: Plasmodium falciparum, 177,000/ $\mu$, sample 2: P. falciparum, 2,500/ $\mu$ l, sample 3: no parasites seen), one didactic sample (Howell-Jolly bodies) and one sample for assessing the quality of staining. Participating laboratories were addressed and selected through the network of the National Tuberculosis Control Programme. Participants were asked to return the responses together with a stained thin and thick blood film for evaluation of Giemsa stain quality.
\end{abstract}

Results: Among 174 participants (response rate 95.1\%), 26.2\% scored samples 1, 2 and 3 correctly and 34.3\%, $21.5 \%$ and $5.8 \%$ of participants reported major errors in one, two or three samples respectively. Major errors included reporting "no malaria" or "non-falciparum malaria" for Plasmodium falciparum-positive samples 1 and 2 (16.1\% and 34.9\% of participants respectively) and "P. falciparum" for Plasmodium negative sample 3 (24.0\%). Howell-Jolly bodies (didactic sample) were not recognized by any of the participants but reported as " $P$. falciparum" by $16.7 \%$ of participants. With parasite density expressed according to the "plus system", $16.1 \%$ and $21.5 \%$ of participants scored one " + " different from the reference score for samples 1 and 2 respectively and 9.7\% and $2.9 \%$ participants scored more than two " + " different. When expressed as counts of asexual parasites/ $\mu$, more than two-thirds of results were outside the mean $\pm 2 \mathrm{SD}$ reference values. The quality of the Giemsa stain was poor, with less than $20 \%$ slides complying with all criteria assessed. Only one quarter of participants purchase Giemsa stain from suppliers of documented reliability and half of participants use a buffered staining solution. One third of participants had participated in a formal training about malaria diagnosis, half of them earlier than 2007.

Conclusion: The present EQA revealed a poor quality of malaria microscopy in DRC.

\section{Background}

The detection of Plasmodium parasites by light microscopy is still the primary method of malaria diagnosis in most health care facilities throughout the world [1]. Quality control programmes are a prerequisite of competent microscopy. The World Health Organization (WHO) recommends the cross-checking of blood slides: a sample of routine blood slides is sent to the reference laboratory, where it is checked for accuracy. External

\footnotetext{
* Correspondence: pgillet@itg.be

${ }^{2}$ Department of Clinical Sciences, Institute of Tropical Medicine (ITM),

Nationalestraat 155 B 2000 Antwerp, Belgium

Full list of author information is available at the end of the article
}

quality assessment (EQA) programmes (also referred to as "proficiency testing") are an alternative approach: in such programmes, the reference laboratory sends stained blood film samples to the peripheral laboratories, which assess them and submit a report, after which they are given feedback about the correct results and their own performance [2].

In the Democratic Republic of the Congo (DRC), malaria is endemic and $97 \%$ of the population is living in areas of stable malaria transmission. Parasite-based diagnosis of malaria is mainly performed by microscopy. Due to difficult economical and logistic conditions, countrywide programmes of quality control of malaria

\section{Biomed Central}

(c) 2011 Mukadi et al; licensee BioMed Central Ltd. This is an Open Access article distributed under the terms of the Creative Commons Attribution License (http://creativecommons.org/licenses/by/2.0), which permits unrestricted use, distribution, and reproduction in any medium, provided the original work is properly cited. 
microscopy do not exist in DRC and till now, EQA programmes on malaria microscopy have been only sporadically organized and were limited to the capital (Kinshasa) [3]. In 2010, the National Malaria Control Programme (Programme National de la Lutte contre le Paludisme, PLNP) organized an EQA session on malaria microscopy through its national reference laboratory (Institut National de Recherche Biomédicale, INRB) and the network of laboratories participating in the national programme of tuberculosis control (Programme National de la Lutte contre la Tuberculose, PNLT). The present study reports the results of this EQA session.

\section{Methods}

Participants

The EQA session was organized in 2010 and addressed clinical laboratories subscribing to the EQA sessions on parasitology of INRB as well as those involved in the network of PNLT as deployed in four provinces: Kinshasa, Bas- Congo, Katanga and the Oriental Province. In addition, the malaria reference laboratories at the provincial level $(\mathrm{n}=11)$ were included. Laboratories were categorized as hospital-based $(n=72)$, referral health centre $(n=36)$, health centre $(n=44)$ and others $(n=11)$. Table 1 gives an overview of the participants who were located in areas of hyper- of holo-endemic malaria transmission, with Plasmodium falciparum accounting for more than $95 \%$ of malaria infections.

\section{Samples}

The EQA panel consisted of five samples. Three samples were prepared as Giemsa-stained thick and thin blood film preparations with accompanying patient's history and white blood cell count/ $\mu$ l (Table 2). Participants were asked to report the diagnosis (malaria yes or no), Plasmodium species identification and parasite density. For parasite density, participants could express the value as asexual parasites/ $\mu \mathrm{l}$ or as a "plus system" scale (range from "+" to " ++++ ") [4,5], as both systems were endorsed by the national malaria control programme at the time of
EQA session. A fourth sample was a thin blood film with red blood cells containing Howell-Jolly bodies, it was considered as a didactic sample. A fifth sample consisted of an unstained thin blood film: participants were asked to stain this blood film according to their usual procedure and return the sample. Finally, participants were asked to select a routinely processed thick blood film slide of their own laboratory and to return it together with the fifth sample. Both samples were used to assess the quality of the staining and thick blood film preparation as performed by the participants.

The samples were prepared from left-overs of routinely drawn EDTA-blood samples obtained from patients presenting at INRB and prepared and processed according to the recommendations of PNLP and the WHO malaria microscopy quality assurance manual $[1,6]$. Briefly, thin and thick blood films of each sample were applied on a single slide (pre-cleaned slides, MenzelGläzer Braunschweig, Germany). Fixation of thin blood films was done with methanol (Panreac, BarcelonaSpain), and thin and thick blood films were stained with Giemsa pH 7.2 (MERCK, Darmstadt- Germany) and examined by light microscopy using a $\times 1,000$ magnification. Parasite densities were estimated by counting asexual parasites against 200 white blood cells in the thick blood film and converting this number to parasites/ $\mu$ l using the actual white blood cell count as calculated microscopically in a Neubauer chamber [7]. To compensate for technical and observational variations in the parasite count, three expert microscopists counted the parasite densities on 10 slides for each Plasmodiumpositive sample, and mean \pm SD counts were calculated. In addition, parasite densities were assessed according to the "plus system", i.e. a semi-quantitative scale estimating the numbers of asexual parasites per high-power fields [5]. Species identification was confirmed by Plasmodium-specific polymerase chain reaction (PCR) $[8,9]$. Stained samples were allowed to dry, packed in plastic boxes and stored for a maximum of 90 days before shipment.

Table 1 Diagnostic laboratories participating in the EQA session on malaria microscopy

\begin{tabular}{lcccccc}
\hline Participants & Bas-Congo & Kinshasa & $\begin{array}{c}\text { Provinces } \\
\text { Province Orientale }\end{array}$ & Katanga & Total & Estimated \% nationwide coverage \\
\hline Provincial Reference Laboratories & 1 & 1 & 1 & 1 & $11^{*}$ & 100,0 \\
Hospitals & 24 & 32 & 4 & 12 & 72 & 9.9 \\
Referral Health Centres & 22 & 11 & 0 & 3 & 36 & $1.0^{* *}$ \\
Health Centres & 6 & 1 & 32 & 5 & 44 & N.D. \\
Private Laboratories & 0 & 10 & 0 & 1 & 11 & 1.9 \\
Total & 53 & 55 & 37 & 22 & 174 & \\
\hline
\end{tabular}

* Including the Provincial Reference Laboratories of the other seven provinces in DRC

** Combined on a total of 8,266 centres

N.D. $=$ no data ${ }^{2}$ 
Table 2 Clinical information, Plasmodium species and parasite density of the samples submitted as part of the EQA session

\begin{tabular}{|c|c|c|}
\hline Sample & Sample information & Reference result/Comment \\
\hline $\begin{array}{l}\text { 1. Thick + thin blood film, } \\
\text { Giemsa stained }\end{array}$ & $\begin{array}{l}\text { Girl } 9 \text { years old, fever, pale, weakness, Leukocyte count } \\
10,400 / \mu l\end{array}$ & $\begin{array}{l}\text { Plasmodium falciparum, parasite density } 177,000 / \mu \mathrm{l} \text { or } \\
\text { " }++++"\end{array}$ \\
\hline $\begin{array}{l}\text { 2. Thick + thin blood film, } \\
\text { Giemsa stained }\end{array}$ & $\begin{array}{l}\text { Woman } 54 \text { years old, headache and fever, Leukocyte count } \\
2,500 / \mu l\end{array}$ & Plasmodium falciparum, parasite density $86 / \mu l$ or "+" \\
\hline $\begin{array}{l}\text { 3. Thick + thin blood film, } \\
\text { Giemsa stained }\end{array}$ & Man 57 years old, check-up visit & No parasites seen \\
\hline $\begin{array}{l}\text { 4. Thin blood film, Giemsa } \\
\text { stained }\end{array}$ & Woman, 39 years old, no clinical information & No parasites seen, presence of Howell-Jolly bodies \\
\hline $\begin{array}{l}\text { 5. Thin blood film, } \\
\text { unstained }\end{array}$ & Man, 36 years old, no clinical information & $\begin{array}{l}\text { Sample to be stained by the participants and returned } \\
\text { for scoring of staining quality }\end{array}$ \\
\hline 6. No sample & $\begin{array}{l}\text { Stained thick blood film sample to be selected from } \\
\text { routine samples by the participant }\end{array}$ & $\begin{array}{l}\text { Sample to be sent to the reference lab for scoring of } \\
\text { staining quality }\end{array}$ \\
\hline
\end{tabular}

\section{Questionnaire}

In addition to the samples, a questionnaire was submitted. The questionnaire addressed the following issues: numbers of requests for malaria diagnosis, slide positive ratio, staining procedures and training. In addition, participants were surveyed about the use of malaria rapid diagnostic tests (RDTs).

\section{Shipments}

Samples and questionnaire were packed in plastic slide boxes (Slide mailer, Menzel-Gläser, Braunschweig, Germany) and shipped in protected envelopes (Air Pro 4, Propac, Malmo, Sweden) according to UN 3373 recommendations (i). For Kinshasa province, envelopes were transported and on-site delivered by car by an INRB collaborator. Samples for the provinces were shipped by private air carrier to the provincial airports (Kisangani, Lubumbashi and Boma/Kimpese in Oriental Province, Katanga and Bas-Congo respectively) where they were received by the provincial coordinators of PNLT or their representatives. Next, they were transported by car and hand-delivered to the participating laboratories. The results and questionnaire forms, as well as the slides of samples 5 and 6 were collected again by the PNLT representative and shipped to INRB.

\section{Validation of samples and questionnaire}

For validation of the samples, shipment and questionnaire, a pilot EQA session was organized among 20 laboratories of known reputation (ten in Kinshasa, four in Bas-Congo, and three in Katanga and Oriental Province each). These 20 participants were actively surveyed about the quality of the samples, instructions and survey. Their results were included in the analysis.

\section{Data entry and analysis}

The results were entered in an Excel database (Microsoft Corporation, Redmond, Washington, USA).
In diagnostic practice in DRC, parasite densities are generally expressed according to the "plus system": therefore, answers in terms of this score were primarily considered. In addition, values expressed as asexual parasites/ $\mu$ l were categorized in comparaison to the mean reference count \pm SD [10]. For evaluation of the quality of staining and sample preparations (Samples 5 and 6), returned samples were assessed by two microscopists and scored according to criteria from previous studies and WHO recommendations (Table 3) [1,11,12]. Discordant results were assessed by a third observer and the consensus result was considered.

Continuous variables were assessed for significance using the Student's t-test. Differences between proportions were tested for significance using the Pearson's Chi-square test or, in case of small sample sizes, a twotailed Fisher's exact test. Trends in proportions were assessed using Chi-square test for trend.

The results for the microscopic diagnosis (samples 1, 2 and 3) were categorized as correct, or with minor and major errors. Major errors included (i) incorrect diagnosis of malaria, i.e. reporting "negative" in the case of a Plasmodium-positive sample and vice versa, (ii) not mentioning the presence of $P$. falciparum (either reporting non-falciparum species in the case of Plasmodium falciparum or no species identification at all) and (iii) parasite densities scored more than two "+" different from the reference result or not scored at all. A minor error was defined as a parasite density differing one "+" from the reference result.

\section{Results}

\section{EQA sessions}

The pilot EQA session was performed between August and September 2010, the regular EQA session was performed during the period September-November 2010. Results of 174 out of 183 laboratories were received, with a response rate of $95.1 \%$. Median duration of shipment from INRB to participants was 9 days (range 1 - 67 days), 
Table 3 Results for the quality of staining of thin and thick blood film samples stained by the participants

\begin{tabular}{|c|c|c|}
\hline Sample & Criteria & $\begin{array}{c}\text { Numbers } \\
(\%)\end{array}$ \\
\hline \multirow[t]{5}{*}{$\begin{array}{l}\text { Sample 5: Thin blood film supplied by EQA, stained by participant and returned to the reference } \\
\text { laboratory }(n=163)\end{array}$} & $\begin{array}{l}\text { No Giemsa stain precipitates } \\
\text { observed }\end{array}$ & $107(65.6 \%$ \\
\hline & Red blood cells stained grey-pink & $147(90.2 \%$ \\
\hline & $\begin{array}{l}\text { Chromatin of lymphocytes stained } \\
\text { purple }\end{array}$ & $60(36.8 \%)$ \\
\hline & $\begin{array}{l}\text { Granules of neutrophils stained } \\
\text { pink }\end{array}$ & $100(61.4 \%$ \\
\hline & $\begin{array}{l}\text { Complies with all criteria } \\
\text { mentioned above }\end{array}$ & $16(9.8 \%)$ \\
\hline \multirow[t]{5}{*}{$\begin{array}{l}\text { Sample 6: Routinely stained thick blood film of the participant submitted to the reference } \\
\text { laboratory }(n=155)\end{array}$} & $\begin{array}{l}\text { Correct dimensions }(>1 \mathrm{~cm}) \text { and } \\
\text { thickness of the film }\end{array}$ & $110(71.0 \%$ \\
\hline & $\begin{array}{l}\text { Complete hemolysis of the red } \\
\text { blood cells }\end{array}$ & $118(76.1 \%$ \\
\hline & $\begin{array}{l}\text { No Giemsa stain precipitates } \\
\text { observed }\end{array}$ & $60(38.7 \%)$ \\
\hline & $\begin{array}{l}\text { Good contrast between nucleus } \\
\text { and cytoplasm }\end{array}$ & $70(45.1 \%)$ \\
\hline & $\begin{array}{l}\text { Complies with all criteria } \\
\text { mentioned above }\end{array}$ & $30(19.4 \%)$ \\
\hline
\end{tabular}

and the reports were returned within a median delay of 4 days (range 1 - 25 days).

\section{Results for the microscopic analysis of the samples}

Tables 4, 5 and 6 present the results for samples 1, 2 and 3 respectively. For sample 1 ( . falciparum, 177.000/ $\mu \mathrm{l}$ ) $59.2 \%$ of participants scored correct results and another $16.1 \%$ reported minor errors, all but one were errors in parasite density (reporting " +++ " instead of the expected "++++" score, $17.8 \%)$. Nearly a quarter $(n=43,24.7 \%)$ of participants reported major errors, these errors included (i) reporting "no malaria" (5.2\% of participants), (ii) "nonfalciparum malaria" (9.8\%), and (iii) "+" or "++"or no parasite density at all (8.6\%); two participants (1.1\%) combined the latter two errors. Sample 2 ( $P$. falciparum, $86 / \mu \mathrm{l})$ was scored correct by less than half $(41.9 \%)$ of participants: 37 (21.5\%) participants reported minor errors, all but one scoring " ++ " for parasite density instead of the expected "+". Sixty-three (36.6\%) of participants reported major errors including (i) "no malaria" (16.3\%), (ii) "non-falciparum malaria" (18.6\%), and parasite densities above "++" (2.9\%). Two-thirds of participants scored sample 3 (no parasites observed) correct. Major errors among the remaining third included reporting of $P$. falciparum malaria $(24.10 \%)$ with $9(5.2 \%)$ of participants reporting parasite densities above "+". None of the participants mentioned the presence of Howell-Jolly bodies in sample 4. By contrast, for this parasite-negative sample, 24 (16.7\%) of participants reported the presence of Plasmodium parasites, mostly $P$. falciparum.

Among the participants who had valid answers for all three samples $(\mathrm{n}=172), 26.2 \%$ scored all three correctly; $34.3 \%, 21.5 \%$ and $5.8 \%$ had major errors for one, two and all three samples respectively.

\section{Parasite density expressed as parasites $/ \mu \mathrm{l}$}

In addition to their report expressed as "plus system", 44 and 41 participants reported parasite densities as

Table 4 Results for sample 1: P.falciparum, parasite density $177.000 / \mu l$ or "++++". Valid answers of 174 participants were included

\begin{tabular}{|c|c|c|c|c|c|c|c|}
\hline \multirow[b]{2}{*}{ Reported result } & \multirow[b]{2}{*}{ No parasites observed } & \multicolumn{5}{|c|}{ Parasite density ("plus system") } & \multirow{2}{*}{$\begin{array}{l}\text { Total } \\
\text { n (\%) }\end{array}$} \\
\hline & & Not reported & + & ++ & +++ & ++++ & \\
\hline P. falciparum & & 1 & 5 & 9 & $27^{* *}$ & $103^{*}$ & $145(83.3 \%)$ \\
\hline P. falciparum and $P$. malariae & & & & & & $1^{* *}$ & $1(0.6 \%)$ \\
\hline P. malariae & & 1 & & & 1 & 2 & $4(2.3 \%)$ \\
\hline No species identification reported & & & & 1 & 3 & 11 & $15(8.6 \%)$ \\
\hline No parasites observed & 9 & & & & & & $9(5.2 \%)$ \\
\hline Total & 9 & 2 & 5 & 10 & 31 & 116 & $174(100 \%)$ \\
\hline
\end{tabular}

*Correct result, including one result reported as " $+++++"$

*** Minor error 
Table 5 Results for sample 2: P.falciparum, parasite density $86 / \mu$ or "+". Valid answers of 172 participants were included

\begin{tabular}{lcccccc}
\hline & \multicolumn{2}{c}{ Parasite density (“plus system") } & \multicolumn{2}{c}{ Total } \\
\cline { 2 - 5 } Reported result & No parasites observed & Not reported & + & ++ & +++ & n (\%) \\
\hline P. falciparum & & 2 & $72^{*}$ & $36^{* *}$ & 3 & $113(65.7 \%)$ \\
P. vivax and P. falciparum & & & $1^{* *}$ & & $1(0.6 \%)$ \\
No species identification reported & 26 & 27 & 5 & $32(18.6 \%)$ \\
No parasites observed & 28 & & 100 & 41 & 3 & $172(100.0 \%)$ \\
\hline Total & & & & $3.1 \%)$ \\
\hline
\end{tabular}

*Correct result

**Minor error

numbers of asexual parasites/ $\mu$ l for samples 1 and 2 respectively. Figures 1 and 2 display the distribution of the parasite densities with respect to the mean $\pm \mathrm{SD}$ reference values. For sample 1, reported parasite densities ranged between 32 and 2,700,000/ $\mu$ l. Thirty-two (72.7\%) participants reported a parasite density > $2 \mathrm{SD}$ above or below the reference value, with 22 (49.9\%) and $1(2.3 \%)$ participants reporting a value 10 times higher or lower the mean reference value. For sample 2, these numbers were 32 (78.1\%), 2 (4.9\%), 16 (39.0\%) respectively, and parasite densities ranged between 1 and $32.000 / \mu \mathrm{l}$.

\section{Quality of Giemsa staining as scored on thin and thick blood film preparations}

Table 3 lists the scores for the quality of the Giemsa staining for sample 5 (thin blood film supplied by EQA) and sample 6 (thick blood film from the participants' routine diagnosis). Although some scores for the individual criteria were high, the overall quality was modest, with only a minority $(<20 \%)$ of returned slides complying with all criteria assessed.

\section{Results of the questionnaire}

One third $(32.7 \%)$ of participants had processed less than 100 samples during the month before the EQA and $58.3 \%$ had processed less than 200 samples. A total of $25(14.9 \%)$ of participants reported a slide positivity ratio below $20 \%$, whereas two-thirds $(66.0 \%)$ declared slide positivity ratios $\geq 40 \%$, among which $26(15.5 \%) \geq$ 80\% (Figure 3).

Nearly two-thirds (63.8\%) of participants had never participated in a formal training on malaria microscopy, and among those who did, more than half were trained earlier than 2007. The situation was worst for the health centres and reference health centres (no training for $69.8 \%$ and $75.0 \%$ of them repectively) than for the Provincial Reference Laboratories and referral hospitals (no training for $45.5 \%$ and $52.5 \%$ of them respectively) but this difference was not statistically significant ( $\mathrm{p}=$ 0.136).

Participants were asked about their supply for Giemsa stain and the use of buffer solution. The vast majority (87.9\%) purchased Giemsa stain as a stock solution. As to the choice of supplier, only one quarter $(25.3 \%)$ of participants procured it from suppliers of documented reliability like central purchasing services, non-governmental organizations and the national malaria control programme, while one third (35.6\%) relied on "fournisseurs ambulants", i.e. private vendors who visit and deliver on site. This reliance was more apparent among the reference health centres $(22 / 36,61.1 \%)$. For preparation of the working solution, half (52.3\%) of participants used a buffer solution, $29.9 \%$ and $17.8 \%$ used distilled water and regular water (mostly tap water) respectively. One quarter $(24.7 \%)$ of participants used RDTs, half of them since one year or less. The majority $(35,81.4 \%)$ used Paracheck (Orchid Biomedical Systems, Goa, India).

Table 6 Results for sample 3: No parasites observed

\begin{tabular}{|c|c|c|c|c|c|c|c|}
\hline \multirow[b]{2}{*}{ Reported result } & \multirow[b]{2}{*}{ No parasites observed } & \multicolumn{5}{|c|}{ Parasite density ("plus system") } & \multirow{2}{*}{$\begin{array}{l}\text { Total } \\
\text { n (\%) }\end{array}$} \\
\hline & & Not reported & + & ++ & +++ & ++++ & \\
\hline No parasites observed & $116^{*}$ & & & & & & $116(66.6 \%)$ \\
\hline P. falciparum & & 1 & 35 & & 4 & 1 & 41 (23.6\%) \\
\hline No species identification reported & & 1 & 11 & 2 & 1 & 1 & $16(9.2 \%)$ \\
\hline P. falciparum, gametocytes & & 1 & & & & & $1(0.6 \%)$ \\
\hline Total & 116 & 3 & 46 & 6 & 2 & 1 & $174(100.0 \%)$ \\
\hline
\end{tabular}

Valid answers of 174 participants included

${ }^{*}$ Correct result 


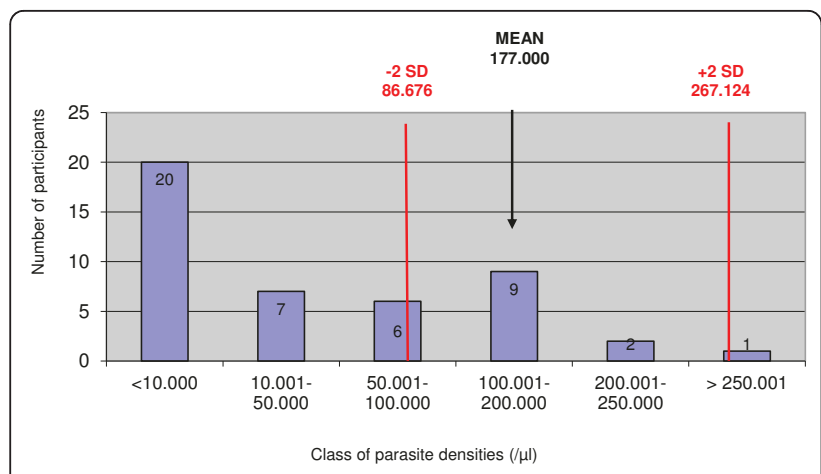

Figure 1 Distribution of parasite densities expressed per $\mu \mathrm{l}$ of blood for sample 1 and $\mathbf{4 4}$ participants. Mean and SD values refer to the expected result as counted by expert microscopy.

\section{Associations between performance and different parameters}

EQA performances in terms of correctness of answers for the three diagnostic samples correlated with the numbers of samples processed monthly $(\mathrm{p}<0.001$, Figure 4) but not with other factors such as province, training during the previous years or quality and origin of the Giemsa stain. In addition, there was a tendency towards better results related to hierarchy of structure, with $16.3 \%$ of health centres, $20.0 \%$ of reference health centres, $34.7 \%$ of hospitals and $45.5 \%$ of provincial laboratories replying correct results for all three samples $(\mathrm{p}=0.064)$.

\section{Discussion}

Among 174 diagnostic laboratories in DRC participating on an EQA session on malaria microscopy, $26.2 \%$ scored all three samples correctly and 34.3\%, 21.5\% and 5.8\% reported major errors in one, two or all three samples respectively. Major errors included missing the diagnosis of $P$. falciparum malaria and diagnosis of malaria in negative samples as well as errors in estimation of parasite density. Most participants had serious problems for

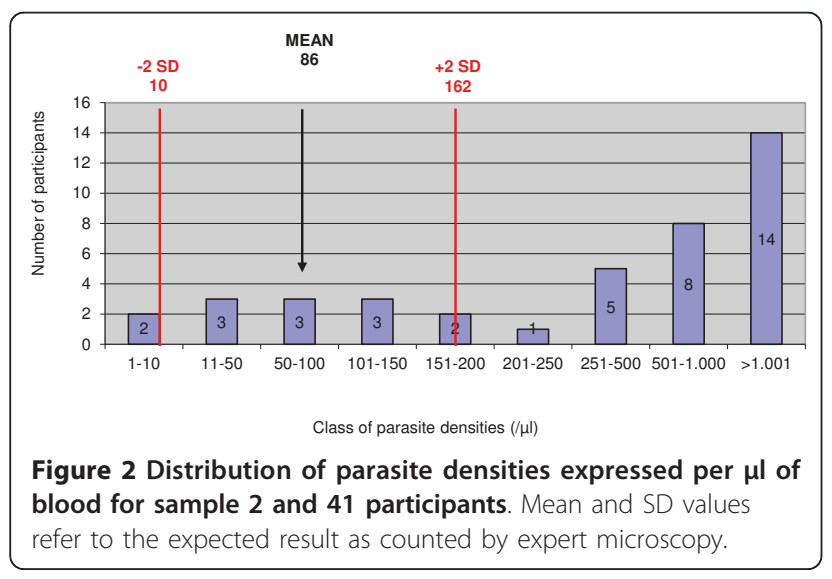

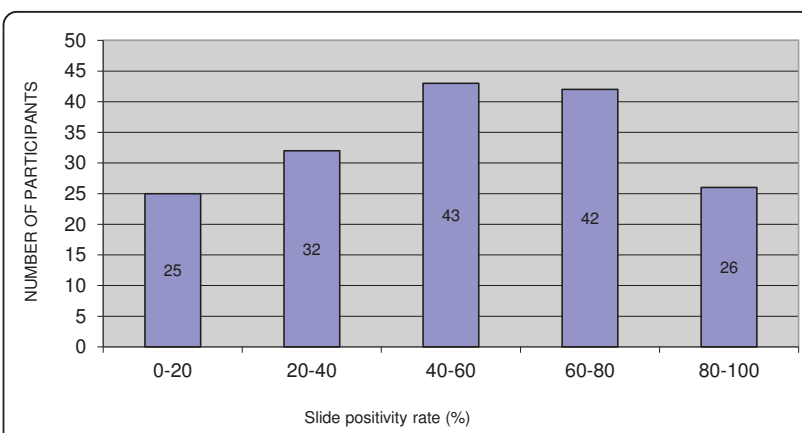

Figure 3 Distribution of slide positive rates among the participants (168 valid answers, data represent numbers).

the purchase and the preparation of the Giemsa stain solution and two-thirds were not formally trained in malaria microscopy.

\section{Errors in diagnosis: false negative and false positive results}

Major errors included reports of "no malaria" or "non-falciparum malaria" in the case of $P$. falciparum-positive samples (16.1\% and $34.9 \%$ for samples 1 and 2 respectively): such false negative results may cause potentially lethal consequences and erode confidence of clinicians and community. Identification up to the level of $P$. falciparum has been noted as a difficulty in non-endemic settings too: a EQA session in Canada in 1995 recorded 27\% errors in the species diagnosis of $P$. falciparum, and $21 \%$ failures were registered at an EQA session in the UK $[12,13]$. Further, $24.0 \%$ and $16.7 \%$ of participants in the present survey reported " $P$. falciparum" in the parasite negative samples 3 and 4 respectively: these false positive results lead to unnecessary treatment adding to costs side effects and distract the clinician from considering other causes of fever and disease. For comparison, EQA sessions in non-endemic

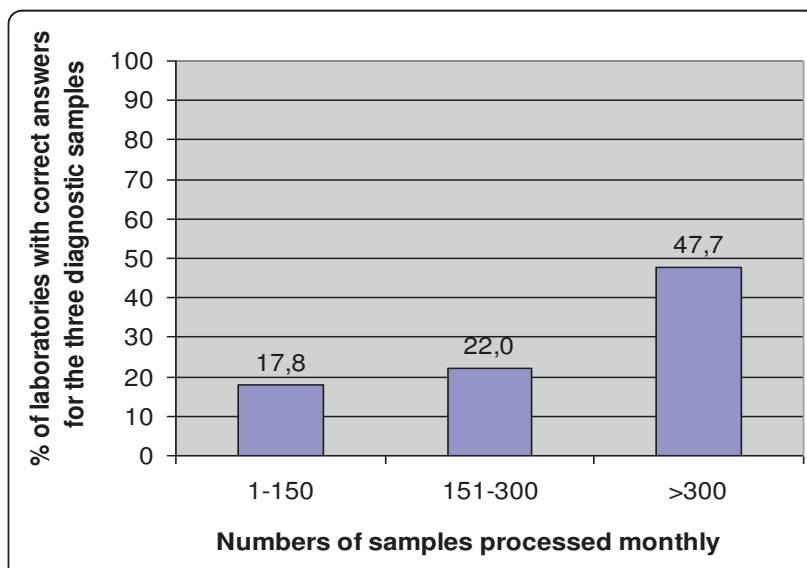

Figure 4 Numbers of samples processed monthly by the participants associated with the percentage of correct answers for the three diagnostic samples. 
sessions recorded false-positive results by $2 \%-15 \%$ of participants and, in line with present observations, HowellJolly bodies, fibrin strands and stain granules were confused with blood parasites [13,14].

\section{Errors in estimation of parasite densities}

With parasite density expressed according to the "plus system", about $20 \%$ and $10 \%$ of participants scored one "+" or $\geq$ "++" different from the reference value (considered as minor and major errors respectively). The quantification according to the "+" scale is subject to inconsistencies and WHO recommends it gradually replacement by counts of asexual parasites/ $\mu$ l. The latter system has only been introduced by PNLP since October 2010, which may explain in part for the poor results presently observed. However, the high numbers of apparent failures (> $10 \times$ above or below the reference value) illustrates the need for clear procedures, training and follow-up during future EQA sessions [10]. Estimation of parasite densities has been revealed as problematic in several EQA sessions in non-endemic settings, with up to $39 \%$ failures $[13,14]$.

\section{Reliable purchase of Giemsa stain and buffer}

The quality of the Giemsa stain was poor, with $<20 \%$ of slides stained by the participants complying with all criteria assessed. Only one quarter of participants purchased Giemsa stain from traceable suppliers such as central purchasing services and the PNLP, while one third relied on private salespersons and only half of them used a buffered solution for the Giemsa stain. These observations may be explained by the difficult economic and logistic situation in DRC. Purchase policies and supplier evaluations are part of all quality assurance systems and essential when considering the Giemsa stain, for which quality-controlled production and $\mathrm{pH}$ monitoring are essential [4].

\section{Training and critical volume}

Only one third of participants had attended a formal training about malaria microscopy, half of them earlier than 2007. Although slightly better at the central and intermediate levels, the situation was of concern too. Training is essential for maintaining competency and commitment of microscopists [4] and PNLP is actually developing trainings programmes. One third of participating laboratories processed less than 100 samples monthly. In addition, there was an association between correct scores for this EQA and the numbers of samples processed monthly. Although the numbers of laboratory staff involved in malaria diagnosis was not accounted, this provides indirect evidence towards the association between low exposure and poorer performance. For microscopists - provided correctly trained - the reading of at least 10 slides a month is recommended by WHO in order to maintain competence [4]. Further, the slide positivity rate varied widely: although till now this parameter was only used as in indirect indicator for malaria transmission and not for laboratory quality assessment $[15,16]$. The value of the slide positivity rate as an indicator of quality assurance may be explored. For instance, slide positivity rates $\geq 80 \%$ even in highly selected patients might be questioned for accuracy [17].

\section{Limitations and strengths of EQA sessions including the present one}

The present EQA session undoubtedly suffered from limitations inherent to EQA methods: for instance, results for samples and questionnaires may reflect theoretical competence and knowledge rather than day-today performance [14]. A second limitation relates to the coverage of this EQA as less than $10 \%$ of hospitals and $1 \%$ of the health centres nationwide were included. Finally, the current participants probably represent the better laboratories, as participation was voluntary and directed to laboratories already involved in the EQA network of the PNLT. Logistic difficulties - a known limitation of EQA sessions in resource constrained settings [18] were behind this selection and approach, since transport infrastructures and internet communication in DRC are limited, and the actual number of participants was only reached by joining the network of PNLT with its regional collaborators.

As to their strengths, it should be noted that EQA sessions particularly when considered in an educational and non-punitive atmosphere offer a didactic stimulus and boost self-confidence and trigger towards implementation of a quality system, as participation is required for accrediting norms such as ISO 15189 for medical laboratories $[2,19]$. For the health authorities and control programmes in resource constrained settings, EQA sessions may be the first or unique information about nationwide laboratory performance, diagnostic practices and the quality of reagents or diagnostic tests [18,20-22]. The results of the present EQA provide benchmark information: this allows to monitor the progress in diagnostic performance through follow-up EQA sessions. In addition, EQA sessions are more cost-effective than crosschecking of slides [18]. Finally, the actual collaboration between the PNLP and PLNT is in line with the WHO recommendation of integrating malaria microscopy quality assessment with that of other microscopically diagnosed diseases such as tuberculosis [23,24].

\section{Place and role of malaria rapid diagnostic test, integration in quality assurance}

The present results are in line with observations about inadequate quality of microscopy in field settings [25]. 
The input needed for corrective actions at all points of microscopy quality assurance will probably be very high; therefore one might tend to favor rolling out malaria rapid diagnostic tests (RDTs) at all levels of health care. Indeed, despite the fact that RDTs no give information about parasite density and species identification, their diagnostic accuracy in field settings may equal or exceed that of microscopy $[26,27]$ and it is obvious that they are less demanding with regard training, equipment and infrastructure. PNLP is deploying RDTs in DRC since June 2010. As for malaria microscopy, malaria RDTs however need to be deployed in a quality assured environment at all levels [28-34].

\section{List of abbreviations}

DRC: Democratic Republic of the Congo; EDTA: Ethylene Diamine Tetraacetic Acid; EQA: external quality assessments; INRB: Institut National de Recherche Biomédicale; ITM: Institute of Tropical Medicine; P: Plasmodium; PCR: polymerase chain reaction; PNLP: Programme National de Lutte contre le Paludisme; PNLT: Programme National de Lutte contre la Tuberculose; RDT(s): Rapid Diagnostic Test(s); SD: standard deviation; UN: United Nations: WHO: World Health Organization.

\section{Acknowledgements}

We would like to thank Dany Nzididi of the parasitology department (INRB, Kinshasa) for technical support and Albert Kanza, Jan Bosco Mayunda, Roger Bakumondo and Niocole Sheshe of the Programme National de Lutte contre la Tuberculose (PNLP, Kinshasa) for the EQA distribution.

\section{Author details}

'Institut National de Recherche Biomédicale (INRB), Kinshasa, Democratic Republic of the Congo. ${ }^{2}$ Department of Clinical Sciences, Institute of Tropical Medicine (ITM), Nationalestraat 155 B 2000 Antwerp, Belgium. ${ }^{3}$ Programme National de Lutte contre le Paludisme (PNLP), Kinshasa, Democratic Republic of the Congo. ${ }^{4}$ Programme National de Lutte contre la Tuberculose (PNLT), Kinshasa, Democratic Republic of the Congo. ${ }^{5}$ Faculté de Médecine Université de Kinshasa, Kinshasa, Democratic Republic of the Congo. ${ }^{6}$ Department of Medical Microbiology, School for Public Health and Primary Care: CAPHRI, Maastricht University Medical Center, The Netherlands.

\section{Authors' contributions}

$P M, P G, A L$ and $B A$ designed the external quality assessment. PM and $A L$ carried out the try-out EQA session. PM, SK and JL carried out the formal EQA session. PM, PG and JJ analyzed and interpreted the results and drafted the manuscript. All authors drafted and critically reviewed the manuscript and approved the final manuscript.

\section{Competing interests}

The authors declare that they have no competing interests.

Received: 22 May 2011 Accepted: 18 October 2011

Published: 18 October 2011

\section{References}

1. World Health Organization: Malaria Microscopy Quality Assurance Manual, version1. 2009 [http://www.searo.who.int/LinkFiles/ Malaria_MalariaMicroscopyManual.pdf].

2. Kettelhut MM, Chiodini PL, Edwards H, Moody A: External quality assessment schemes raise standards: evidence from the UKNEQAS parasitology subschemes. J Clin Pathol 2003, 56:927-932.

3. Mulumba M, Muyembe JJ: Diagnostic de l'infection palustre à Kinshasa, contrôle de qualité inter et intralaboratoires. Congo Médical 1999, 11:759-770.

4. World Health Organization: Basic Malaria Microscopy - Part II.Tutor's Guide., 22010 [http://www.searo.who.int/LinkFiles/ Malaria_malaria_microscopy_Tutors_guide2010.pdf].
5. World Health Organization: Basic Malaria Microscopy - Part I.Learner's Guide., 22010 [http://www.searo.who.int/LinkFiles/

Malaria_malaria_microscopy_Learners_guide2010.pdf].

6. PNLP: Manuel de formation sur le diagnostic de laboratoire du paludisme. 2010.

7. World Health Organization: Manual of basic techniques for a health laboratory., 22003 [http://hinfo.humaninfo.ro/gsdl/healthtechdocs/ documents/s16537e/s16537e.pdf].

8. Cnops L, Jacobs J, Van Esbroeck M: Validation of a four-primer real-time PCR as a diagnostic tool for single and mixed Plasmodium infections. Clin Microbiol Infect 2010.

9. Cnops L, Van Esbroeck M, Bottieau E, Jacobs J: Giemsa-stained thick blood films as a source of DNA for Plasmodium species-specific real-time PCR. Malar J 2010, 9:370.

10. Trudel L, Turcotte P: Rapport d'activités 2007-2008 du laboratoire de Santé Publique du Québec: External quality control blood parasitology. 2008 [http://www.inspq.qc.ca/pdf/publications/790_rapport_activites_lspq. pdf].

11. Dini L, Frean J: Quality assessment of malaria laboratory diagnosis in South Africa. Trans R Soc Trop Med Hyg 2003, 97:675-677.

12. Milne LM, Kyi MS, Chiodini PL, Warhurst DC: Accuracy of routine laboratory diagnosis of malaria in the United Kingdom. J Clin Pathol 1994, 47:740-742.

13. Thomson S, Lohmann RC, Crawford L, Dubash R, Richardson H: External quality assessment in the examination of blood films for malarial parasites within Ontario, Canada. Arch Pathol Lab Med 2000, 124:57-60

14. Kettelhut MM, Chiodini PL, Edwards H, Moody A: External quality assessment schemes raise standards: evidence from the UKNEQAS parasitology subschemes. J Clin Pathol 2003, 56:927-932.

15. Jensen TP, Bukirwa H, Njama-Meya D, Francis D, Kamya MR, Rosenthal PJ, Dorsey G: Use of the slide positivity rate to estimate changes in malaria incidence in a cohort of Ugandan children. Malar J 2009, 8:213.

16. Reyburn H, Mbatia R, Drakeley C, Carneiro I, Mwakasungula E, Mwerinde O, Saganda K, Shao J, Kitua A, Olomi R, Greenwood BM, Whitty CJ: Overdiagnosis of malaria in patients with severe febrile illness in Tanzania: a prospective study. BMJ 2004, 329:1212.

17. Petti CA, Polage CR, Quinn TC, Ronald AR, Sande MA: Laboratory medicine in Africa: a barrier to effective health care. Clin Infect Dis 2006, 42:377-382.

18. Libeer J, Richerdson H, Bullock D, Heuck C, Tholen D, Martin R, Carter J, Boone E, Noble M: Addressing issues associated with the development and management of PT programs and with their optimum use. Accred Qual Assur 2002, 7:320-334.

19. Tholen D: Improvements in performance in medical diagnostics tests documented by interlaboratory comparison programs. Accred Qual Assur 2002, 7:146-152.

20. Gillet P, Mukadi P, Vernelen K, Van Esbroeck M, Muyembe JJ, Bruggeman C, Jacobs J: External quality assessment on the use of malaria rapid diagnostic tests in a non-endemic setting. Malar J 2010, 9:359.

21. Snell Jj: United Kingdom National External Quality Assessment Scheme for Microbiology. Eur J Clin Microbiol 1985, 4:464-467.

22. Snell JJ, Supran EM, Tamashiro H: WHO international quality assessment scheme for HIV antibody testing: results from the second distribution of sera. Bull World Health Organ 1992, 70:605-613.

23. Sarkinfada F, Aliyu Y, Chavasse C, Bates I: Impact of introducing integrated quality assessment for tuberculosis and malaria microscopy in Kano, Nigeria. J Infect Dev Ctries 2009, 3:20-27.

24. World Health Organization: Malaria light microscopy. Creating a culture of quality 2005 [http://www.wpro.who.int/internet/resources.ashx/MVP/ malaria_light_microscopy.pdf].

25. Alonso PL, Barnwell JW, Bell D, Hanson K, Mendis K, Moonen B Newman RD, de Savigny D, Schapira A, Slutsker L, Tanner M, Teuscher T: A research agenda for malaria eradication: diagnoses and diagnostics. PLoS Med 2011, 8:e1000396.

26. Batwala V, Magnussen P, Nuwaha F: Are rapid diagnostic tests more accurate in diagnosis of plasmodium falciparum malaria compared to microscopy at rural health centres? Malar J 2010, 9:349.

27. de Oliveira AM, Skarbinski J, Ouma PO, Kariuki S, Barnwell JW, Otieno K, Onyona P, Causer LM, Laserson KF, Akhwale WS, Slutsker L, Hamel M: Performance of malaria rapid diagnostic tests as part of routine malaria case management in Kenya. Am J Trop Med Hyg 2009, 80:470-474. 
28. World Health Organization: How to use a Rapid Diagnostic Test (RDT): A guide for training at a village and clinic level 2008 [http://www.wpro.who.int/ sites/rdt/using_rdts/training/rdt_training_falciparum.htm].

29. World Health Organization: Tranporting, Storing and Handling Malaria Rapid Diagnostic Tests at central and peripheral storage facilities 2009 [http:// deliver.jsi.com/dlvr_content/resources/allpubs/guidelines/ TranStorRDT_Central.pdf].

30. World Health Organization: Malaria Rapid Diagnostic Test Performance; Results of WHO product testing of malaria RDTs: Round 1 (2008) 2009 [http:// www.finddiagnostics.org/resource-centre/reports_brochures/malariadiagnostics-report-2009.html]

31. World Health Organization: Overview of the prequalification of diagnistics assessment process 2010 [http://www.who.int/entity/diagnostics_laboratory/ evaluations/pqdx_007_pq_overview_document_v3.pdf].

32. World Health Organization: Information note on interim selection criteria for procurement of malaria rapid diagnostic tests (RDTs) 2010 [http://www.who. int/entity/malaria/diagnosis_treatment/diagnosis/infoRDTinterimcriteria.pdf].

33. World Health Organization: Lot-testing: Using quality control parasite dilutions to test the sensitivity of malaria RDTs 2010 [http://www.wpro.who.int/sites/ rdt/using_rdts/qa/lot_testing.htm].

34. World Health Organization: Good practices for selecting and procuring rapid diagnostic tests for malaria 2011 [http://whqlibdoc.who.int/publications/ 2011/9789241501125_eng.pdf].

doi:10.1186/1475-2875-10-308

Cite this article as: Mukadi et al: External quality assessment of malaria microscopy in the Democratic Republic of the Congo. Malaria Journal 2011 10:308.

\section{Submit your next manuscript to BioMed Central} and take full advantage of:

- Convenient online submission

- Thorough peer review

- No space constraints or color figure charges

- Immediate publication on acceptance

- Inclusion in PubMed, CAS, Scopus and Google Scholar

- Research which is freely available for redistribution

Submit your manuscript at www.biomedcentral.com/submit 